\title{
Research on the Development Trend and Countermeasures of the New Business Mode of Traditional Chinese Medicine Health Tourism
}

\author{
Zhenyu $\mathrm{Hu}$ \\ School of Economics and Management \\ Jiangxi University of Traditional Chinese Medicine \\ Nanchang, China
}

\author{
Quan Xia \\ School of Economics and Management \\ Jiangxi University of Traditional Chinese Medicine \\ Nanchang, China
}

\author{
Zhiwang Ding * \\ Education management \\ Jiangxi University of Traditional Chinese Medicine \\ Nanchang, China \\ *The corresponding author
}

\begin{abstract}
As an emerging industry with broad prospects, TCM Health Tourism has gradually become a new format of TCM culture and tourism industry. This paper has made an in-depth explanation of it from the aspects of government guidance, characteristic drive, supporting construction, brand building, bottlenecks and shortcomings, etc., in order to promote the rapid development of Chinese medicine health tourism industry and boost the vigorous development of the comprehensive health industry.
\end{abstract}

Keywords-TCM health tourism; new business mode; countermeasure research

\section{INTRODUCTION}

People's health is a significant mark for a prosperous nation and a powerful country. A moderately prosperous society in all respects cannot be built without a healthy nation. With the development of the economic society and the improvement in living standards, people emphasize more on their health. As health tourism has gradually emerged in the common life of the public, the TCM health tourism industry is now thriving. Developing a new business mode featuring "TCM plus health tourism" is conducive to promoting the development of TCM and the big health industry. It also becomes an indispensable driving force of the development of the tourism industry. The new business mode can continuously satisfy the people's pursuit of a better life, and will give strong impetus to the future economy.

\section{BRight FeAtures of the New Business Mode OF Traditional Chinese Medicine Health TOURism}

As an emerging derivative industry, TCM health tourism integrates health, tourism, culture, leisure, sports, health care, diet and entertainment, and involves a variety of industries such as medicine, health, tourism, health, catering, education and services. It is both an important part of the health tourism industry and a new business mode that shows the

This paper is a phased achievement of the Research on the Cultivation of Innovation and Entrepreneurship of College Students of Traditional Chinese Medicine in the Humanities and Social Science Project of Jiangxi Provincial Department of Education (JY18124). characteristics of TCM. Due to its unique connotation and distinctive characteristics, it has become a sunrise industry with great development potential and a new attractive business mode. "TCM plus health tourism" has combined traditional Chinese medicine with health tourism, health preservation and healthy living. It helps promote the in-depth integration of TCM health services and tourism, and injects new impetus to the development of the TCM industry and tourism. During their travels, tourists will secure individual development and good moods, acquire the knowledge about TCM health preservation, experience TCM cultural products, and keep in a robust and healthy physical condition.

\section{A. Achieving the Organic Combination of Traditional Chinese Medicine and Health Tourism}

As a branch of ecological tourism, TCM health tourism is an unexplored integrated industry that combines tourism and TCM, and marks an extension of TCM and tourism. Based on traditional tourism services and the cultural philosophy of TCM, TCM health tourism has integrated TCM and tourism by allowing tourists to visit TCM historical culture, appreciate Chinese herbs and experience TCM products, forming a TCM health tourism mode featuring TCM health services, travel, leisure, health care, recovery, fitness and entertainment.

\section{B. Achieving the Organic Combination of Traditional Chinese Medicine and Health Preservation}

TCM is a treasure of the Chinese nation, with profound health preservation knowledge. Through TCM health tourism, tourists can get involved in the long-standing TCM culture and the health preservation knowledge, understand the health concept carried by TCM culture, and directly learn the health preservation knowledge of TCM. During their travels, consumers will get both physical and mental relief, and gain knowledge of health preservation, rehabilitation and prevention of illnesses such as cardiovascular and cerebrovascular diseases, hypertension and diabetes, thus advocating the public to keep in good health. 


\section{Achieving the Organic Combination of Traditional Chinese Medicine and Healthy Living}

TCM health tourism breaks through a single travelling way, and merges tourism, leisure and healthy living together. It is a new tourism service mode that integrates recuperation, recovery, visits and tours. Through health travels of TCM, consumers not only enhance their physical conditions, but also obtain the knowledge about TCM including healthy living, diet therapy and physiotherapy, which is beneficial to enhance people's understanding of healthy living, help them adapt to external environments, and reduce or prevent the occurrence of diseases.

\section{BRoAd Prospects of the New Business Mode of Traditional Chinese Medicine Health Tourism}

With profound TCM culture, diverse cultural resources, various Chinese herbs, long-standing processing techniques, and multiple health care methods, the "TCM plus health tourism" business mode embraces tremendous market prospects.

\section{A. Huge Market, Broad Prospects}

According to a research report released by the Stanford Research Institute, the number of global medical health travelers has increased from 20 million in 2006 to 40 million in 2012. In recent years, this trend continues to gain momentum. According to a forecast made by the World Health Organization, the medical health-related service industry will become the world's largest industry by 2020, and leisure tourism-related services will rank second. The two industries will account for 22\% [1] of global GDP. Facing the vast medical tourism market, TCM health tourism has rapidly emerged nationwide, gradually becoming a new trend of national tourism and a new economic engine. In the global medical tourism market which is now thriving, China's traditional medicine health tourism should go up with the trend and accelerate development, thus gaining a share in the global medical tourism market.

\section{B. Strong Needs, Massive Potential}

With the accelerated pace of modern urban lives, increased work pressures and unhealthy lifestyles, the sub-health problem becomes increasingly serious. The "Big Health Data of Chinese People" issued in 2014 pointed out that $75.7 \%$ of white-collar workers in China's major cities are in sub-healthy conditions, and $60 \%$ of them are in overworked conditions. TCM health tourism emerges as a new healthy business mode that meets people's needs for a healthy and good life. According to the "2016 Online Medical Tourism Report" released by the Ctrip, an online travel agency, around 500,000 Chinese people went abroad for medical services in 2016. The number of Chinese tourists that sought for overseas medical travel through the Ctrip was 5 times that of the previous year. The per capita expenditure exceeded 50,000 yuan (\$7243 USD), about 10 times that of outbound travel[2]. The huge health needs have opened up a broad market space for the TCM health tourism industry. As a new business mode that aims to satisfy people's pursuit of physical and mental health, TCM health tourism boasts bright market trends and development prospects.

\section{Plentiful Resources, Unique Advantages}

China is home to abundant natural tourism resources such as springs, forests and islands, cultural tourism resources including Confucianism, Buddhism and Taoism, diverse Chinese herbal resources with an increasing planting scale, featured herb processing resources, and local TCM culture in Anhui, Guangdong, Jiangsu, Henan and Jiangxi. After in-depth cultivation, these varied and featured natural and cultural resources can become distinctive and advantageous cultural resources featuring TCM health tourism. China's unique and various TCM cultural resources can develop into TCM natural tourism, TCM historical tourism and TCM demonstration zone tourism centering around sightseeing, or cultural experience tourism centering around individual experience such as Chinese herbal bath, herbal plantation and recognition and medicated diet making, or cultural tourism centering around festival exhibitions such as TCM cultural festivals, TCM utensils, TCM painting and calligraphy exhibitions, or TCM hot spring tourism and health preservation tourism centering around health maintenance and health care. Additionally, various TCM cultural bases, industrial parks, historical museums have provided strong supports for the TCM health tourism industry.

\section{National Support, Numerous Preferential Policies}

With the rapid development of the big health industry, China has attached unprecedented importance to the TCM health tourism industry, and successively issued a series of policy documents including the " 'Healthy China 2030' Planning Outline", the "Opinions on Promoting the Development of Health Service Industry", the "Opinions on Promoting the Development of Traditional Chinese Medicine Health Tourism", the "Development Plan for Traditional Chinese Medicine Health Service (2015-2020)", the "Notice on the Establishment of National Traditional Chinese Medicine Health Tourism Demonstration Zones (Bases, Projects)" and the "Strategic Plan for the Development of Traditional Chinese Medicine (2016-2030)". In about 3 years, China plans to build 10 national TCM health tourism demonstration zones, 100 national TCM health tourism demonstration bases, and 1,000 national TCM health tourism demonstration projects across the country. In September 2017[3], the National Tourism Administration and the National Administration of Traditional Chinese Medicine authorized 15 units as the National Demonstration Zones for TCM Health Tourism. In March 2018, the National Tourism Administration and the National Administration of Traditional Chinese Medicine also announced the first batch of 73 units as the first batch of national demonstration bases for TCM health tourism, of which Anhui, Jiangxi and Shandong boast four units respectively[4].

\section{E. Local Support, Strong Momentum}

With the great supports of national policies, local governments also attach great importance to the development of TCM health tourism. Many provinces and cities have proactively responded to national policies by exploring the development of TCM health tourism and putting forward numerous policies and industry planning that benefit TCM health tourism. The local governments are very optimistic 
about the promising TCM health tourism industry. Many provinces and cities have successively issued a series of policy documents to promote the development of TCM health tourism. TCM health tourism is becoming a new potential sector, a new mode in the tourism industry and a driving force for the development of the tourism industry. In 2018, Shandong, Hebei, Henan, Anhui, Guangdong and other provinces successively approved the first batch of units authorized to establish provincial-level TCM health tourism demonstration zones (bases and projects). The units included areas and enterprises with a high concentration of medicine, health and tourism such as Chinese medicine hospitals, tourism demonstration areas, Chinese medicine museums and pharmaceutical groups. Under the active leadership of the Ministry of Culture and Tourism and National Administration of TCM, TCM health tourism is developing in a stable method in numerous areas.

\section{Developmental Bottlenecks And ShORTCOMINGS ANALYSIS OF THE NEW BUSINESS MODE OF Traditional ChINESE MEDicine HEALTH TOURISM}

Driven by the "Healthy China Initiative" and supported by the country and local governments, the new business mode of TCM health tourism continues to boom and maintain a strong momentum. However, if we want to further develop the new mode, equip it with Chinese characteristics and local advantages, build a TCM health tourism brand, and make it an important engine to drive the development of the big health industry and tourism industry in the new era, we will find a number of developmental bottlenecks and shortcomings.

\section{A. Inadequate Top-level Planning and Design, Incomplete Industrial System}

As an emerging business mode, TCM health tourism covers many industries, and features a long industrial chain along with complex and diverse elements. It involves TCM historical resources, cultural resources, educational resources, tourism resources and ecological resources. The new business mode faces numerous problems such as insufficient top-level design and incomplete industrial system. The development process lacks high-end planning, and features weak comprehensive competitiveness, inadequate link points, feeble industrial cluster effects, small industrial scale and narrow radiation range. The new mode has not formed economies of scale and has not leveraged the benefits of industrial clusters and brand effects. The related sectors involved in TCM health tourism have not established corresponding market participation, ecological compensation, and benefit-sharing mechanisms. The participants in the market is not enthusiastic enough to exert the maximum value of the resources, which is not conducive to the extension and expansion of the TCM health tourism industry. As an effective coordinated mechanism has not be established, the parties involved in the new mode are in lack of cooperation, and the market concentration rate is obviously low. To develop and advance the TCM health tourism industry, it is necessary to strengthen the top-level design and planning, and integrate various resources and development elements such as TCM, tourism, health, culture, education, science and technology according to the characteristics of the industries and projects, thus forming an industrial chain featuring diverse and connected business modes.

\section{B. Insufficient Resource Development, Inadequate Capital Investment}

TCM boasts profound culture, abundant and various resources, and the TCM industry and health care technologies show their distinctive features, but these resources are in shortage of integration and in-depth development. The TCM health tourism industry is still small in scale and weak in competitiveness and has not seen a distinctive and robust well-known brand.

(i) Inadequate expansion of featured resources development. TCM resources and tourism resources have not secured enough integrated development. Large comprehensive tourism projects combing medicine, nursing, travel, entertainment and catering have not be established. The radiation effects have not been leveraged and the joint value of featured TCM resources and cultural tourism resources has not been widely evacuated.

(ii) Inadequate depth of featured resources development The homogenization of tourism products and service projects is serious. Local featured elements have not been fully integrated. In-depth development has not been carried out. Local featured cultural landscapes and the advantages of TCM historical culture have not been highlighted. These products and projects are short of characteristics and differentiated experiences. They cannot meet the needs of different groups of people.

(iii) Inadequate efforts in featured resources development Shandong, Hebei, Henan, Sichuan and Jiangxi are all actively building TCM health tourism scenic areas, but many of them are established on the basis of previous tourist attractions. Some projects are directly dependent on Chinese medicine hospitals, causing insufficient featured integration, simple TCM cultural atmosphere, and less attraction to consumers.

(iv) Inadequate capital investment. Limited by insufficient understanding of the new business mode of TCM health tourism and weak demonstration and radiation effects of the industrial development, the government and market entities fail to establish a sound coordinated mechanism, an effective market participation mechanism and a profit distribution mechanism, causing ineffective combination between the industry and capital and poor connectivity with the Internet finance. Problems like inactive participation of market entities, insufficient capital investment, and lagged capital investment still remain obvious in some areas.

\section{Imperfect Coordinated Development Mechanism,} Incomplete Industrial Supplementary Facilities

The TCM health tourism industry involves a wide range of industries and requires supporting systems and coordination of other parties. However, problems like insufficient coordinated development and industrial supports still exist in development.

(i) Imperfect coordinated development mechanism. The administrative departments of TCM, tourism, culture and other related industries belong to different administrative agencies. Although the development is led by cultural tourism and TCM 
management departments, the coordinated promotion of administrative services is still vulnerable because the TCM health tourism industry covers a wide range of sectors. The phenomenon, therefore, impairs the enthusiasm of market entities and leads to insufficient capital investment, which directly limits the development and the expansion and development of the new business mode.

(ii) Imperfect protective development mechanism. As an emerging industry, the TCM health tourism industry still needs to be enhanced in inviting investment, media publicity, brand promotion and support services. It also needs to be standardized in protection, guidance, supervision and service. In terms of market access, there are still no effective measures and specific laws and regulations. The management services of TCM health service projects are not standardized. Blind pursuit of economic benefits even prevails in some areas. The behavior wastes resources destroys the natural environments in scenic areas, disrupts the order in scenic areas, and causes redundant construction and malicious competition, restricting the development of the industry[6].

(iii) Inadequate industrial supports. During the promotion of the TCM health tourism industry, the collaborative cultural innovation, tourism services, hotel services, catering services, health care services, and TCM health services are still imperfect, which makes it difficult to meet the individualized needs of consumers for TCM tourism. Asymmetrical information between upstream and downstream industries and tangled links cannot help achieve an industrial system of health tourism services with distinctive TCM features.

(iv) Inadequate human resources. At present, a large number of tourism management and TCM professionals remain active in the talent market, but the composite talents with relevant professional skills and management capabilities are seriously inadequate. Those who are skilled in TCM culture and good at tourism management, human resources, marketing promotion and scenic area operation are desperately needed. In addition, professional requirements have not been set for related employees, places, environments, equipment and facilities. Job seekers can be employed as long as they secure relevant certificates. Strict requirements for their professional background and qualifications are still blank[5].

\section{Inadequate TCM Features, Weak Brand Effect}

China boasts profound TCM culture and unique TCM health tourism resources. The development of the TCM tourism industry gains strong momentum. However, China's TCM health tourism industry is still at the preliminary stage. Problems like unclear TCM characteristics, mediocre highlights and low market share still lie in many aspects such as featured projects, product management, brand image building and leading business cultivation. Although there is a lack of TCM health tourism in the overseas medical tourism industry, foreign medical tourism boasts diverse items, sound industry reputation, successful integration between the medical industry and the tourism industry and pleasant travel experience. Different development modes of medical tourism such as South Korean beauty, Thai SPA and Indian health tourism hospital have become local pillar industries, international medical brands and a new growth point for the local economy. These industries have greatly driven the development of tourism, created brilliant economic benefits, and advanced economic and social development. Compared with South Korea, Thailand, and India, China's medical tourism industry still has a huge gap, and has not formed TCM health tourism brands with core competitiveness. China's Hainan and Guangdong are integrating medical care, physical therapy and TCM resources by supporting leading businesses, expanding reputation and influence of brands, and following a development path of TCM health tourism centered on the featured brand operation. The medical tourism industry in South Korea, Thailand and India has provided a good inspiration and reference for the development of China's TCM health tourism. In order to improve the featured development of TCM health tourism, efforts must be stepped up in enhancing featured products management, publicity and marketing, as wells as setting typical examples and leading brands.

\section{ENHANCING FEATURES, StimUlating Vitality, AND PRomoting THE SOUND DEVELOPMENT OF THE NEW Business MODE OF TCM HEALTH TOURISM}

The new business mode of TCM health tourism has promising development potential, broad markets and development prospects. In order to promote the rapid rise of the TCM health tourism industry and make it a new engine to drive the development of the big health industry, it is necessary to strengthen government guidance, optimize market allocation, improve development mechanism, accelerate supporting construction, enhance featured engine, emphasize brand building, and leverage the advantages of the Internet plus new media.

\section{A. Enhancing Government Guidance, Strengthening Market Allocation}

Promoting the development of the TCM health tourism industry requires coordinated cooperation of various parties. In the development of the TCM health tourism industry, government departments should strengthen the top-level design and high-end planning, and actively play the leading role of policies and overall planning by improving systems and standards. The departments should formulate practical measures in macro guidance, policy-driven development and industrial layout, and make more efforts in investment introduction, preferential tax and resource development so as to create a sound business environment for TCM health tourism, exert the decisive role of the market in resource allocation, and stimulate the enthusiasm of various parties and market entities.

(i) Macro-control and incentive support. Related supporting policies should be formulated to form an incentive mechanism that is designed to promote the development of the TCM health tourism industry. The government should support local businesses by ensuring their land usage, enhancing investment and financing guidance, and improving fiscal and taxation price policies. The government should proactively guide the integrated development of the TCM industry and tourism industry by further simplifying approval procedures for projects, relaxing market access, and establishing open and transparent access systems. 
(ii) Scientific planning and innovative development. A featured development path combining TCM, culture, tourism, leisure, catering and entertainment should be followed. The spatial layout of the industrial development and the role of functional areas should be properly arranged so as to create comprehensive composite projects featuring TCM culture, travel and elderly care. A competitive mechanism should be introduced to cultivate market entities along with influential and competitive leading businesses. Efforts should be made to broaden development and utilization channels and find new competitive and profitable ways. The construction of industrial clusters should be advanced to enhance the supports for related industries.

(iii) Open wider and promote consumption. The government should proactively develop upstream and downstream industries related to TCM health tourism, set different consumption levels for different consumer groups, expand consumption levels, consumption ranges and the number of consumers in order to upgrade the consumption. Efforts should be made to further open the market, connect overseas tourists markets, and put forward a series of preferential policies on visa, air tickets and medical insurance for overseas tourists. The personal economic interests and personal property of tourists should be guaranteed so that international consumers can be attracted to China.

\section{B. Strengthening the cultivation of innovative Chinese medicine and entrepreneurial talents to meet the needs of Chinese medicine for healthy tourism}

Promote the development of TCM health tourism industry, use China's unique TCM resources to play the role of the market, strengthen the training needs of TCM innovation and entrepreneurship services, and promote the combination of TCM and health tourism, so as to solve the shortage of talents in China's current TCM health tourism industry phenomenon.

(i)Cultivate social adaptation skills through humanities literacy and Chinese medicine courses and extracurricular activities to form vocational basic skills. It is required to complete basic professional experiments and basic professional skills in the laboratory and on-campus training base to form professional general skills;

(ii)To construct a scientific and rational modern Chinese medicine medical service Chinese medicine health tourism service curriculum system. It is required to complete on-the-job internships and practice in off-campus enterprises to form comprehensive application capabilities to meet the needs of healthy tourism.

\section{Accelerating Supporting Construction, Improving Guarantee Mechanisms}

The TCM health tourism industry is a new sustainable business mode that goes with the national trend of industrial structural up-gradation and economic transformation. It features long economic value chain and low energy consumption pollution. The TCM health tourism industry, a comprehensive industrial project integrating health care, leisure, tourism and entertainment, involves supports from hotels, catering, transportation, insurance, tourists attractions, travel agencies and other related industries. The TCM health tourism industry, therefore, requires protection, supervision and coordination of numerous parties.

(i) Accelerating supporting facilities and improve infrastructure. Corporations with substantial capitals and overall competitive edges should be introduced to participate in the development and construction of tourism projects as well as improve tourism facilities and services. Preferential policies and conditions should be provided to sectors to attract a large amount of social funds and shape a sound development atmosphere. Supervision should be enhanced and the guarantee mechanism should be improved.

(ii) Enhancing supervision and standardizing industrial operation. Efforts should be made to improve related management and supervision mechanisms for TCM health tourism, formulate guidelines for TCM health tourism demonstration zones, establish a sound quality standard, management system and supervision system for the TCM industry and tourism industry, revise a series of laws and regulations that suit TCM health tourism such as tourism management regulations and protection regulations. Industry standards and market access rules related to TCM health tourism should be formulated. The standard certification system of the service industry should be normalized. Measures must be taken to crack down on illegal false propaganda and illicit commercial practice. Efforts must be stepped up to supervise and give feedback on the practical progress to ensure all works are implemented.

(iii) Enhancing IT construction with the help of Internet Plus. In order to improve tourists' travel experience, it is necessary to actively promote the development of new information technologies such as high-tech media technologies, cloud computing, Internet of Things, block chains and mobile Internet, establish a tourism information network covering all tourist attractions, and build a comprehensive service information platform for TCM health tourism including functions like archives information, multilingual consultation and reservation management. Efforts should be made to advance research and development, put forward smart tourism products, and introduce VR technologies so that tourists can experience the profound cultural connotations of TCM and quality modern information service provided by scenic areas[7].

\section{Enhancing Feature-driven Development, Advancing Brand Building}

Relevant parties should make full use of the natural TCM resources in regions, give full play to the featured advantages of TCM culture, and merge them with TCM historical and cultural resources. Based on protection and recycling, relevant parties should put units featuring prominent TCM elements together such as TCM medical institutions, TCM health care institutions, Chinese herbal medicine manufacturers, planting bases for quality medicinal materials, medicinal botanical gardens, historic sites, hot springs and scenic areas. Additionally, a batch of TCM health tourism businesses (bases) and TCM health tourism complex featuring distinctive characteristics and obvious advantages should be constructed. 
(i) Stressing TCM cultural elements and enhancing features of health tourism. Businesses should dig deeper into the cultural elements of TCM, and integrate local cultural elements of TCM with tourism products, so that the products and services feature clear cultural background and the cultural connotations of TCM products can be elevated. Relevant departments should develop brand demonstration zones and demonstration bases in terms of culture, ecology, sports, diet, experience, etc., creating distinctive brands and routes for TCM health tourism. The promotional function of the media should be fully played to ensure the brand features and the healthy image of TCM health tourism can be effectively advertised. It is necessary to cooperate with travel agencies and design a large number of TCM health tourism routes through the market-oriented operation.

(ii) Strengthening publicity and planning and increasing brand awareness. Relevant departments should initiate targeted publicity activities themed around TCM health tourism, and carry out online and offline multi-channel marketing and publicity using the Internet so as to enhance the popularity of tourism brands. Relevant departments should set a high-end orientation, enhance publicity, elevate the grade of scenic areas, optimize the structure of products, and build high-quality brands. Relevant departments should determine proper images for major TCM cultural tourist attractions, leverage the advantages of tourism resources, and combine local featured resources with personal needs, thus winning the trust of the public and shape distinctive brands. Efforts should be made to determine a proper market orientation, and ensure the strategic collaboration among prices, channels and promotion so that the brands can be more resilient and influential[8].

(iv) Following a differentiated development path according to different local conditions. Treatment, rehabilitation and tourism should be integrated and featured services should be provided according to the developmental features and tourism advantages of local TCM. Emphasis should be placed on the cultivation of tourism projects and products centering on health services and cultural experiences. The effective and in-depth development of superior TCM resources should be achieved through tourism. Personalized and diverse health tourism products should be guaranteed to meet people's various needs. The prices of the products should be reasonable and different pricing strategy should be adopted to avoid the undesirable influences caused by price wars on TCM health tourism brands. Process control should be emphasized in terms of the services provided in order to improve visitors' experiences and win a good reputation.

\section{CONCLUSION}

The key to developing Chinese medicine health tourism industry is to strengthen government guidance and talent cultivation. Only when the government speeds up the support and the introduction of guarantee mechanisms, as well as brand building, can we truly deliver high-quality service talents for the rapid development of China's traditional Chinese medicine health tourism industry, in order to meet the development needs of the society for the new business mode of Chinese medicine health tourism industry.

\section{REFERENCES}

[1] Liu Yurong. Chinese medicine health tourism should play "Chinese characteristics" [N]. China Youth Daily. 2017-04-20 (6)

[2] 2016 Online Medical Tourism Report [R] http://www.chinanews.com/life/2016/12-29/8108331.html

[3] Two units in Gansu were selected as the first batch of national TCM health tourism demonstration bases. People's Network http://gs.people.com.cn/n2/2018/0413/c183283-31457373.htm

[4] Chinese medicine health tourism will become the market new blue sea [EB/OL].Http://www.ce.cn/culture/gd/201512/07/t20151207_7349420. html

[5] Yan Yi. Research on the supervision of TCM health care service institutions [J]. Management of Traditional Chinese Medicine, 2017, (7) 48-50.

[6] Cheng Yong, Shi Yun, Cai Yuming. Discussion on the development of Chinese medicine health care service industry [J]. Management of Traditional Chinese Medicine, 2011, 19 (7).

[7] Peng Yuling, Wang Lei, Xia Yumei. A preliminary study on the supply-side structural reform of the Chinese medicine industry in the era of "Internet + great health" [J]. Health Economics Research, 2018, 375(7): 35-36.

[8] Rao Zhihua, Zhai Zhiwei. Developing Jiangxi cultural industry brand in participating in the Belt and Road Initiative [J]. Open Guide, 2018, (1) 10 